\section{Fístula coronaria gigante. Presentación clínica, caracterización angiográfica, tratamiento y seguimiento a largo plazo. Caso clínico}

\author{
HÉCTOR UGALDE, DIEGO UGALDE, GASTÓN DUSSAILLANT
}

\section{Giant coronary artery fistula. Report of one case}

Coronary artery fistulae are abnormal connections between a coronary artery and any cardiac chamber or other vessels. Most of them have a congenital origin. We report a 60 years old woman referring a history of progressive dyspnea and orthopnea during the last year. A continuous heart murmur was audible in the third and fourth intercostal spaces at the left sternal border. Electrocardiogram was normal and echocardiography showed mild dilation of right cavities and an image suggesting a dilated right coronary artery with flow to right atrium. Coronary angiography was performed, showing a normal left coronary artery and a very large, tortuous right coronary artery with an extensive communication to coronary venous sinus. Surgical treatment was decided and was performed without incidents. The patient is well after five years of follow up.

(Rev Med Chile 2016; 144: 941-945)

Key words: Arteriovenous Fistula; Coronary Vessels; Coronary Vessel Anomalies.

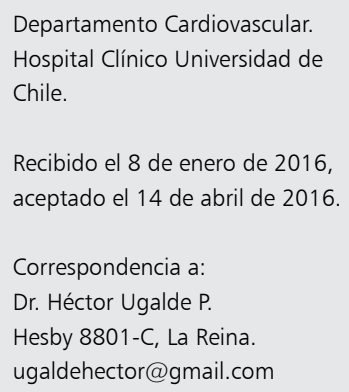

L as anomalías de las arterias coronarias son de infrecuente presentación e incluyen variaciones en el origen, en su calibre, en su trayecto y en su lugar de finalización ${ }^{1}$. Dentro de ellas están las fístulas coronarias, que se definen como conexiones anormales entre una arteria coronaria y una cámara cardiaca o un vaso intraaórtico mayor, la mayor parte de ellas de origen congénito ${ }^{2,3}$, pero, en algunas ocasiones pueden ser adquiridas $^{4-6}$. Su significación clínica no esta muy bien estudiada, se considera que la mayor parte de ellas no presentan síntomas, pero en algunos casos pueden ocasionar síntomas de isquemia o insuficiencia cardiaca ${ }^{7}$ y también ser objeto de complicaciones como endocarditis bacteriana, disección, trombosis e infarto al miocardio ${ }^{8,9}$. El objetivo de esta presentación es mostrar la presentación clínica, angiográfica, el tratamiento y la evolución a largo plazo en un paciente con fístula coronaria congénita gigante, primero de estos casos descritos en el país y en base a este caso revisar el tema en relación a esos puntos mencionados.

\section{Caso clínico}

Paciente mujer de 60 años sin factores de riesgo cardiovascular, ni antecedentes de patologías o intervenciones previas, asintomática y sin utilización de fármacos quien 2 años antes del diagnóstico inicia cuadro caracterizado por sensación de falta de aire con esfuerzos moderados, por lo que inicialmente consulta a médico general quien encuentra un examen físico normal, luego solicita exámenes generales de laboratorio, los cuales (hemograma, perfil bioquímico, examen de orina y electrocardiograma) son normales, con estos resultados indica medidas generales (restricción 


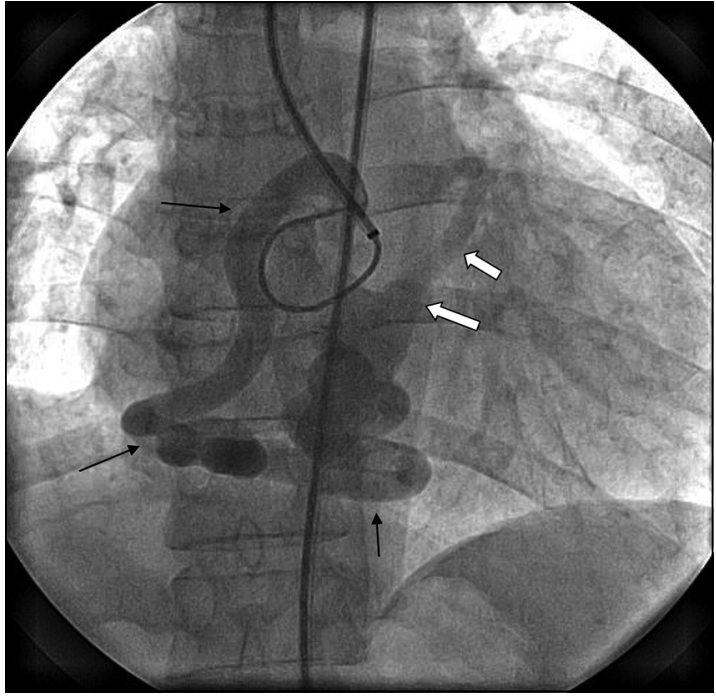

Figura 1. Coronaria derecha dilatada, tortuosa $(\rightarrow)$ que drena en forma amplia en seno coronario $(\Rightarrow)$, no visualizándose sus ramas (RM, DP ni RV).

sal y esfuerzo físico) con lo cual la sintomatología no cambia. Ante esto solicita un ecocardiograma el cual fue informado como completamente normal, por lo cual insiste en las medidas generales y agrega un diurético en dosis bajas, a pesar de lo cual no se observa mejoría. Posteriormente se agrega ortopnea y discreta mayor limitación física, asociado a palpitaciones rápidas de corta duración, ocasionales. Por este motivo la paciente decide consultar a especialista en cardiología quien detecta en el examen físico un soplo continuo en tercer y cuarto espacio paraesternal izquierdo de baja intensidad. El resto del examen es completamente normal. Ante este hallazgo solicita un nuevo ecocardiograma en nuestra institución, el cual muestra dilatación leve de cavidades derechas e imagen de coronaria derecha dilatada con probable flujo desde distal a cavidad auricular derecha. Por aumento de sintomatología se define estudio angiográfico, el cual muestra arteria coronaria izquierda normal y una arteria coronaria derecha (ACD) de gran tamaño, trayecto muy tortuoso y que drena en forma amplia en el seno coronario (Figura 1). En la Figura 2 podemos ver una ACD normal y compararla con la que presenta nuestra paciente. Se discute el caso solicitando la opinión de cardiólogo intervencional pediátrico, y se define que debido al tamaño de la fístula la mejor opción

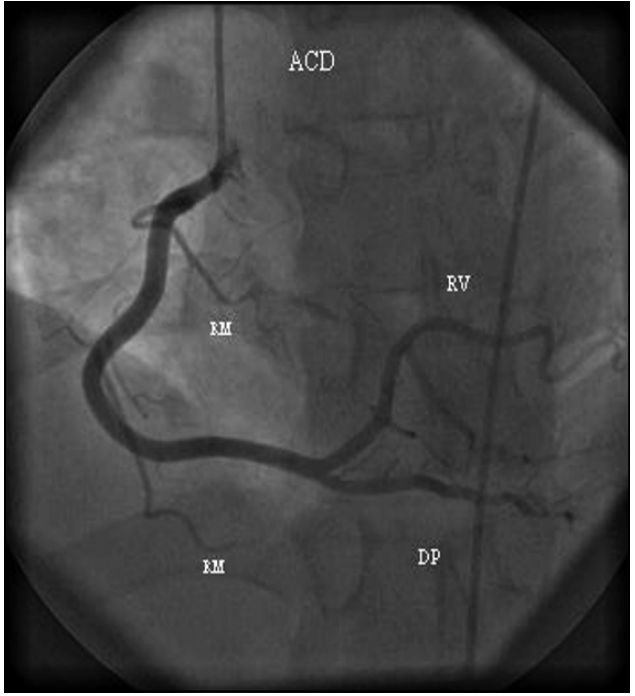

Figura 2. Arteria coronaria derecha (ACD) normal con sus ramas marginales (RM) y ramas terminales, las arterias descendente posterior (DP) y retroventricular (RV).

es el tratamiento quirúrgico. En la cirugía se efectúa cierre de fístula con sutura directa, evolución post operatoria sin incidentes. Posterior a la cirugía, dentro de la misma hospitalización se realiza un control angiográfico, el cual muestra cierre completo de la fístula. La paciente evoluciona en buenas condiciones, a los 5 años, asintomática, sin eventos.

\section{Discusión}

Este caso nos muestra a un paciente adulto con una fístula coronaria congénita gigante sintomática, lo que constituye un hecho excepcional. La presencia de fístulas coronarias es muy baja, se describe entre 0,2 a $0,6 \%$ en series angiográficas $^{10-12}$, de estas la mayor parte son de origen congénito $^{2,3}$, pero también pueden ser adquiridas, como puede ocurrir en pacientes trasplantados sometidos a repetidas biopsias coronarias $\mathrm{s}^{4,5} \mathrm{u}$ otros traumas como angioplastía coronaria, marcapaso ventricular y cirugía cardiaca, entre otros ${ }^{13}$.

La mayor parte de las fístulas coronarias congénitas son pequeñas y no sintomáticas ${ }^{3,10,14}$ y se presentan como una anomalía aislada ${ }^{15}$, aunque más excepcionalmente pueden asociarse a otras anomalías congénitas como nacimiento anómalo ${ }^{16}$ o aneurismas coronarios ${ }^{17}$, su descubrimiento 
ocurre en general en forma casual en el desarrollo de un ecocardiograma o en una angiografía coronaria solicitados por otra razón que la misma fístula o ante la sospecha clínica a través de la presencia de un soplo y el estudio consiguiente de este. La aparición de fístulas coronarias gigantes como la de nuestra paciente es aún más infrecuente, no se conoce su real prevalencia, y sólo se describen casos aislados en la literatura ${ }^{9,18}$.

En nuestro país este sería el primer caso descrito, y es más raro aun llegar a la vida adulta sin haber sido detectada, pues suelen producir síntomas a diferencia de las demás.

La evolución natural de las fístulas coronarias es poco conocida, se describe un bajo porcentaje de cierre espontáneo, $1-2 \%{ }^{19,20}$, la mayoría se mantiene en el tiempo y algunas de ellas pueden crecer en el tiempo hasta llegar a producir sobrecarga de volumen y falla cardiaca como suponemos ocurrió en el caso descrito, o producir isquemia miocárdica por robo coronario. Otras, aun sin crecer pueden ser fruto de complicaciones, como trombosis en el vaso nativo, aneurisma y ruptura o endocarditis bacteriana entre ellas s,8,9,13-15 $^{2,}$

Los síntomas más comunes que se presentan en esta anormalidad son disnea y dolor toráci$\mathrm{Co}^{13,15,21}$, y estos síntomas suelen ocurrir en fístulas de tamaño grande al menos, como nuestra paciente que consultó por disnea de esfuerzo. El diagnóstico preliminar de esta entidad suele plantearse ante la presencia de un soplo cardíaco, el cual se describe en $82 \%$, en los pacientes adultos que han llegado a estudio ${ }^{15}$, y se puede confirmar con un ecocardiograma, especialmente en edad pediátrica, pero también en pacientes adultos ${ }^{2,3}$. La necesidad de angiografía en el estudio es más bien para delinear la verdadera anatomía y definir las alternativas terapéuticas en el paciente ${ }^{2,14,15,21}$, aunque en el adulto puede ser requerida como método diagnostico, en nuestro caso el ecocardiograma sugirió el diagnóstico y la angiografía lo confirmó. También, en el último tiempo, el diagnóstico puede también ser confirmado por tomografía coronaria ${ }^{22}$. El tratamiento de las fístulas coronarias es aun controversial. Algunos apuntan al cierre de toda fístula encontrada, independiente de su tamaño o de si produce síntomas ${ }^{8,23}$. Esto, como ya se comentó, basados en la posibilidad de desarrollar alguna complicación.

Otros opinan lo contrario ${ }^{2,3,21}$, de acuerdo con lo propuesto por las guías de manejo de las cardiopatías congénitas publicadas por la sociedad americana de cardiología ${ }^{24}$, que preconiza el cierre de fístulas sintomáticas o que produzcan alguna complicación y las fístulas grandes, independiente de su sintomatología. En este caso en particular, con una fístula muy grande y sintomática está claramente indicado su cierre.

Para el cierre definitivo de una fístula coronaria, el método inicial descrito fue la cirugía, existen muchos casos y series de casos así tratados ${ }^{23,25}$, pero desde un tiempo ha adquirido relevancia el cierre percútaneo, efectuado con diferentes dispositivos, y también existe abundante literatura que preconiza esta forma de tratar las fístulas coronarias ${ }^{26,28}$.

Tanto uno como el otro presentan algunas ventajas y también algunas complicaciones, y es importante recordar que ningún tratamiento invasivo, por sencillo que parezca descrito, está exento de posibles complicaciones como embolización del material, infarto al miocardio, vasoespasmo $^{26,28}$ o la posibilidad tardía de reaparición de la fístula ${ }^{26}$ para el caso del cierre percútaneo o infarto al miocardio, complicaciones asociadas a la intervención misma y reaparición nuevamente de la fístula para la cirugía ${ }^{8,25}$.

Sea cual sea la forma escogida, los resultados descritos son buenos, con baja morbimortalidad, y la evolución a largo plazo, cuando ha sido consignada, es similar ${ }^{22}$. La decisión va a depender de la experiencia de los equipos y de la anatomía de la fístula. En caso de fístulas gigantes, como esta, los pocos casos descritos han sido tratados con cirugía ${ }^{9,18}$ como nosotros lo hicimos. La evolución con cirugía es buena, con baja morbimortalidad ${ }^{22,25}$, como en este caso y a largo plazo el pronóstico es adecuado, lo cual también ocurrió con nuestra paciente, quien mejoró su sintomatología y permanece en buenas condiciones, sin eventos cardiovasculares a 5 años de su tratamiento.

\section{Conclusión}

Se muestra el caso de una paciente con una fístula coronaria gigante; la fístula coronaria en sí es de baja prevalencia en estudios angiográficos, pero la incidencia de fístulas coronarias como esta es desconocida, sólo hay casos aislados publicados. Estos son siempre sintomáticos y requieren tratamiento definitivo, la mayor parte de las veces con cirugía como así ocurrió, con buenos resultados iniciales y a largo plazo. 


\section{Referencias}

1. Normal and anomalous coronary arteries: Definitions and classification. Angelini P. Am Heart J 1989; 117: 418-34.

2. Hauser M. Congenital anomalies of the coronary arteries. Heat 2005; 1240-45.

3. Webb G, Mallhorn J, Therrien J, Redington A. Cap. 62: Congenital Heart Disease. En: Libby P, Bonow R, Mann D, Zipes D. Braunwald's Heart Disease, 10th ed. Saunders Elsevier; Philadelphia 2015. p. 1443.

4. Sandhu J, Uretsky B, Zerbe T, Goldsmith A, Reddy P, Kormos $\mathrm{R}$, et al. Coronary artery fistula in the heart transplant patient. A potential complication of endomyocardial biopsy. Circulation 1989; 79: 350-6.

5. Marti V, Bailen J, Auge J, Bordes R, Crexells C. Fístula coronaria a ventrículo derecho en pacientes trasplantados cardíacos como complicación de las biopsias. Rev Esp Cardiol 1991; 44: 320-3.

6. Spaedy T, Wilensky R. Coronary artery fistulas: clinical implications. ACC Current Journal Review 1994; 3: 24.

7. Angelini P, Velasco J, Flamm S. Coronary anomalies. Incidente, Pathophysiology, and Clinical Relevance. Circulation 2002; 105: 2449-54.

8. Liberthson R, Sagar K, Berkoben J, Weintraub R, Levine F. Congenital coronary arteriovenous fistula. Report of 13 patients, review of the literature and delineation of management. Circulation 1979; 59: 849.

9. Sáez de Ibarra J, Fernández-Tarrío R, Forteza F, Bonnín O. Giant coronary artery fistula between the left main coronary artery and the superior vena cava complicated by coronary artery dissection. Rev Esp Cardiol 2010; 63: 743-4.

10. Yamanaka O, Hobbs R. Coronary artery anomalies in 126.595 patients undergoing coronary arteriography. Catheterization and Cardiovascular Diagnosis 1990; 21: 28-40.

11. Barriales R, Morís C, López A, Hernández L, San Román $\mathrm{L}$, Barriales V, et al. Anomalías congénitas de las arterias coronarias del adulto descritas en 31 años de estudios coronariográficos en el Principado de Asturias: principales características Angiográficas y clínicas. Rev Esp Cardiol 2001; 54: 269-81.

12. Ugalde H, Ramírez A, Dussaillant G, Ayala F, García S, Silva AM, et al. Angiografía coronaria: indicación, resultados y complicaciones en 5.000 pacientes consecutivos. Rev Med Chile 2007; 135: 829-38.

13. Said S, El Gamal M, Vander Werf T. Coronary Arteriovenous Fistulas: Collective Review and Management of Six New Cases-Changing Etiology, Presentation, and Treatment Strategy. Clin Cardiol 1997; 20: 748-52.
14. Qureshi S. Review: Coronary arterial fistulas. Orphanet Journal of Rare Diseases 2006; 1: 51.

15. Said S. Current characteristics of congenital coronary artery fistulas in adults: A decade of global experience. World J Cardiol 2011; 26; 3(8): 267-77.

16. Koneru J, Samuel A, Joshi M, Hamden A, Shamoon F, Bikkina M. Coronary anomaly and coronary artery fistula as cause of angina pectoris with literature review. Case Rep Vasc Med 2011; 486-87.

17. Hirose H, Amano A, Yoshida S, Nagao T, Sunami H, Takahashi A, et al. Coronary artery aneurysm associated with fistula in adults: collective review and a case report. Ann Thorac Cardiovasc Surg 1999; 5 (4): 258-64.

18. Maleszka A, Kleikamp G, Minami K, Peterschröder A, Körfer R. Giant coronary arteriovenous fistula. A case report and review of the literature. Z Kardiol 2005; 94 (1): 38-43.

19. Said S, Lam J, Van der Werf T. Solitary coronary artery fistulas: a congenital anomaly in children and adults. A contemporary review. Congenit Heart Dis 2006; 1 (3): 63-76.

20. Hackett D, Halliedie-Smith K. Spontaneous closure of coronary artery fistula. Br Heart J 1984; 52: 477-9.

21. Gowda R, Vasavada B, Khan I. Review: Coronary artery fistulas: Clinical and therapeutic considerations. International Journal of Cardiology 2006; 107: 7-10.

22. Said S, Nijhuis R, Op den Akker J, Kimman G, Van Houwelingen K, Gerrits D, et al. Diagnostic and therapeutic approach of congenital solitary coronary artery fistulas in adults: Dutch case series and review of literature. Neth Heart J 2011; 19: 183-91.

23. Tirolomis T, Aleksic I, Bush T, Zenker D, Rushewski W, Daichau H. Congenital coronary artery fistulas in adults: surgical treatment and outcome. Int J Cardiol 2005; 98 : 57-9.

24. ACC/AHA 2008. Guidelines for the Management of Adults With Congenital Heart Disease: A Report of the American College of Cardiology/American Heart Association Task Force on Practice Guidelines (Writing Committee to Develop Guidelines on the Management of Adults With Congenital Heart Disease): Developed in Collaboration With the American Congenital Heart Disease, Society for Cardiovascular Angiography and Interventions, and Society of Echocardiography, Heart Rhythm Society, International Society for Adult Congenital Heart Disease, Society for Cardiovascular Angiography and Interventions, and Society of Thoracic Surgeons Circulation 2008; 118: e714-e833.

25. Lowe J, Oldham H, Sabiston D. Surgical Management of Congenital Coronary Artery Fistulas. Annals of Surgery 1981; 194; 4: 373-80. 
26. Jama A, Barsoum M, Bjarnason H, Holmes D, Rihal C. Percutaneous closure of Congenital Coronary Artery Fistulae. Results and Angiograpfic Follow-up. J Am Coll Cardiol Intv 2011; 4: 814-21.

27. Díaz L, Fournier J, Gómez S, Mayol A, González A, Pérez J. Fístulas de arterias coronarias en adultos. Oclusión percutánea mediante coils. Rev Esp Cardiol 2005; 58 (1): 93-6.

28. Armsby L, Keane J, Sherwood M, Forbess J, Perry S, Lock J. Management of Coronary Artery Fistulae. Patient Selection and Results of Transcatheter Closure. J Am Coll Cardiol 2002; 39: 1026-32. 